\title{
A closed form solution for pollutant dispersion in atmosphere considering nonlocal closure of the turbulent diffusion
}

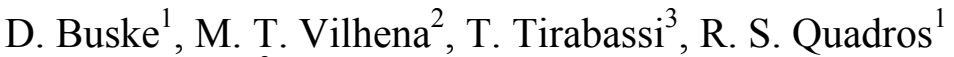 \\ \& B. Bodmann ${ }^{2}$ \\ ${ }^{1}$ Universidade Federal de Pelotas (UFPel), Pelotas (RS), Brazil \\ ${ }^{2}$ Universidade Federal do Rio Grande do Sul (UFRGS), \\ Porto Alegre (RS), Brazil \\ ${ }^{3}$ Institute of Atmospheric Science and Climate (ISAC-CNR), \\ Bologna, Italy
}

\begin{abstract}
Atmospheric air pollution turbulent fluxes can be assumed proportional to the mean concentration gradient. This assumption, along with the equation of continuity, leads to the advection-diffusion equation. Moreover, large eddies are able to mix scalar quantities in a manner that is counter to the local gradient. In this work we present an analytical solution of the three-dimensional steady state advection-diffusion equation, considering nonlocal turbulence closure using the Integral Transform Technique (GILTT). Numerical results and statistical comparisons with experimental data are presented.

Keywords: air pollution modelling, analytical solutions, advection-diffusion equation, nonlocal closure, integral transform.
\end{abstract}

\section{Introduction}

In the last years, special attention has been given to the issue of searching analytical solutions for the advection-diffusion equation in order to simulate the pollutant dispersion in the Atmospheric Boundary Layer (ABL). We are aware of the existence of analytical solutions in the literature, but for specific and particular problems. Among them we mention the works [1-6]. In fact, all these solutions are valid for very specialized practical situations with restrictions on 
wind and eddy diffusivities vertical profiles. Costa et al. [7] presented a semianalytical solution of the multidimensional advection-diffusion equation for more realistic physical scenario using an integral formulation. The solution is valid for a limited ABL and general wind and eddy diffusivity vertical profiles, but expressed by a stepwise function $[8,9]$

Finally a general two-dimensional solution without any restriction in the spatial function of wind and eddy diffusion coefficients was presented by [10, 11]. The solving methodology was the Generalized Integral Laplace Transform Technique (GILTT) that is an analytical series solution including the solution of an associate Sturm-Liouville problem, expansion of the pollutant concentration in a series in terms of the attained eigenfunction, replacement of this expansion in the advection-diffusion equation and, finally, taking moments. This procedure leads to a set of differential ordinary equations that is solved analytically by Laplace transform technique. A complete review of the GILTT method is given in [12]. More recently the three-dimensional GILTT solution (3D-GILTT) considering local closure of the turbulence was presented by Buske et al. [13]. The idea of solution is the application of the integral transform in the y-direction and then the resultant two-dimensional problem is solved following the previous works. No approximation is made along the solution derivation so that is an exact solution except for the round-off error.

In this work we take a step forward assuming a three-dimensional problem with nonlocal closure of the turbulent diffusion. The problem of closing the turbulence in the advection-diffusion equation is modified considering a generic equation for the turbulent diffusion. The countergradient term in the turbulence closure made additional terms to appear in the advection-diffusion equation and these terms are related to the asymmetrical transport in the convective boundary layer. This new equation is solved by the 3D-GILTT method. Numerical results and statistical comparisons with experimental data are presented.

\section{The advection-diffusion equation and the 3D-GILTT method}

The advection-diffusion equation of air pollution in the atmosphere is essentially a statement of conservation of the suspended material and it can be written as:

$$
\bar{u} \frac{\partial \bar{c}}{\partial x}+\bar{v} \frac{\partial \bar{c}}{\partial y}+\bar{w} \frac{\partial \bar{c}}{\partial z}=-\frac{\partial \overline{u^{\prime} c^{\prime}}}{\partial x}-\frac{\partial \overline{v^{\prime} c^{\prime}}}{\partial y}-\frac{\partial \overline{w^{\prime} c^{\prime}}}{\partial z}+S
$$

where $\bar{c}$ denotes the average concentration of a passive contaminant $\left(\mathrm{g} / \mathrm{m}^{3}\right)$, $\bar{u}, \bar{v}, \bar{w}$ are the mean wind $(\mathrm{m} / \mathrm{s})$ components along the axis $x, y$ and $z$, respectively and $S$ is the source term. The terms $\overline{u^{\prime} c^{\prime}}, \overline{v^{\prime} c^{\prime}}, \overline{w^{\prime} c^{\prime}}$ represent, respectively, the turbulent fluxes of contaminants $\left(\mathrm{g} / \mathrm{sm}^{2}\right)$ in the longitudinal, crosswind and vertical directions.

Observe that eqn. (1) has four unknown variables (the concentration $\bar{c}$ and turbulent fluxes) which lead us to the known turbulence closure problem. One of the most widely used closures for eqn. (1), is based on the gradient transport 
hypothesis (or K-theory) which, in analogy with the Fick's law of molecular diffusion, assumes that turbulence causes a net movement of material down the gradient of material concentration at a rate which is proportional to the magnitude of the gradient [14]. So:

$$
\overline{u^{\prime} c^{\prime}}=-K_{x} \frac{\partial \bar{c}}{\partial x} ; \overline{v^{\prime} c^{\prime}}=-K_{y} \frac{\partial \bar{c}}{\partial y} ; \overline{w^{\prime} c^{\prime}}=-K_{z} \frac{\partial \bar{c}}{\partial z}
$$

where $\mathrm{K}_{\mathrm{x}}, \mathrm{K}_{\mathrm{y}}, \mathrm{K}_{\mathrm{z}}$ are the Cartesian components of eddy diffusivity $\left(\mathrm{m}^{2} / \mathrm{s}\right)$ in the $x, y$ and $z$ directions, respectively. In the first order closure all the information on the turbulence complexity is contained in the eddy diffusivities.

The eqn. (2), combined with the continuity equation of mass, leads to the advection-diffusion equation. For a Cartesian coordinate system we rewrite the advection-diffusion equation like [15]:

$$
\bar{u} \frac{\partial \bar{c}}{\partial x}+\bar{v} \frac{\partial \bar{c}}{\partial y}+\bar{w} \frac{\partial \bar{c}}{\partial z}=\frac{\partial}{\partial x}\left(K_{x} \frac{\partial \bar{c}}{\partial x}\right)+\frac{\partial}{\partial y}\left(K_{y} \frac{\partial \bar{c}}{\partial y}\right)+\frac{\partial}{\partial z}\left(K_{z} \frac{\partial \bar{c}}{\partial z}\right)+S
$$

The simplicity of the K-theory of turbulent diffusion has led to the widespread use of this theory as mathematical basis for simulating pollutant dispersion (open country, urban, photochemical pollution, etc.). But K-closure has its own limits. In contrast to molecular diffusion, turbulent diffusion is scaledependent. This means that the rate of diffusion of a cloud of material generally depends on the cloud dimensions and the intensity of turbulence. As the cloud grows, larger eddies are incorporated in the expansion process, so that a progressively larger fraction of turbulent kinetic energy is available for the cloud expansion.

Another problem is that the down-gradient transport hypothesis is inconsistent with observed features of turbulent diffusion in the upper portion of the mixed layer, at convective cases where countergradient material fluxes are known to occur [16]. Because countergradient fluxes are thought to be indicative of boundary layer scale eddies, as opposed to small scale ones, such fluxes are often called non-local fluxes. Local K-theory is a method for parameterize the effects of turbulent mixing based on how small eddies will mix quantities along a local gradient of the transported quantity

Already some decades ago it was noted that in the upper part of convectively driven boundary layers, the flux of scalars are counter to the gradient of the mean scalar profile [17]. The mean potential temperature gradient and the flux change sign at different levels introducing a certain region in the convective boundary layer where they have the same sign. This was in contrast with the common view in first order turbulent closure that turbulent diffusion is down gradient. In order to describe diffusion also in these regions, Ertel [18] and Deardoff [17, 19] proposed to modify the usual applied flux-gradient relationship in K-theory approach according to

$$
\overline{w^{\prime} c^{\prime}}=-K_{z}\left(\frac{\partial \bar{c}}{\partial z}-\gamma\right)
$$

where $\gamma$ represents the countergradient term. 
Many schemes and parameterization for counter-gradient term have been developed in literature. Here we use the parameterization proposed by van Dop and Verver [20] which is based on the work of Wyngaard and Weil [21]:

$$
\left[1+\left(\frac{S_{k} T_{L_{w}} \sigma_{w}}{2}\right) \frac{\partial}{\partial z}\right] \overline{w^{\prime} c^{\prime}}=-K_{z} \frac{\partial \bar{c}}{\partial z}
$$

where $\mathrm{S}_{\mathrm{k}}$ is the skewness of the vertical turbulent velocity (w'), that is, $S_{k}=$ $\overline{w^{3}} /\left(\overline{w^{\prime 2}}\right)^{3 / 2}, \sigma_{w}$ is the vertical turbulent velocity standard deviation $(\mathrm{m} / \mathrm{s})$ and $T_{l w}$ is the Lagrangian time scale $(s)$. The second term in the operator (in the brackets) represents the nonlocal counter-gradient term.

Using eqns. (4) and (5) the turbulence closure problem is solved without obey the Fick's law, being called non-Fickian closure (known also as nonlocal closure). The non-Fickian closure allows the investigation of more energetic eddies in different heights and the effect of the asymmetric transport in the computation of the pollutant concentration considering in a more complete way the structure of the turbulent dispersion.

Applying the above equations in (1) the advection-diffusion equation, in the Eulerian framework for a Cartesian coordinate system in which the $\mathrm{x}$ direction coincide with that of the average wind, is written as:

$$
\bar{u} \frac{\partial \bar{c}}{\partial x}=K_{y} \frac{\partial^{2} \bar{c}}{\partial y^{2}}+\frac{\partial}{\partial z}\left(K_{z} \frac{\partial \bar{c}}{\partial z}\right)-\frac{\partial}{\partial z}\left(\beta \bar{u} \frac{\partial \bar{c}}{\partial x}\right)+\frac{\partial}{\partial z}\left(\beta K_{y} \frac{\partial^{2} \bar{c}}{\partial y^{2}}\right)
$$

where $\beta=0.55 \mathrm{~S}_{\mathrm{k}} \sigma_{\mathrm{w}} \mathrm{T}_{\mathrm{lw}}$, for $0<\mathrm{z}<\mathrm{h}, 0<\mathrm{y}<\mathrm{Ly}$ and $\mathrm{x}>0$. In this work we neglect the diffusion component $K_{x}$ because we assume that the advection is dominant in the x-direction and also consider that $K_{y}$ has only dependence on the z-direction. Equation (6) is subjected to the boundary conditions:

$$
\begin{aligned}
K_{z} \frac{\partial \bar{c}}{\partial z}=0 & \text { at } z=0, h \\
K_{y} \frac{\partial \bar{c}}{\partial y}=0 & \text { at } y=0, L_{y}
\end{aligned}
$$

and to the source condition:

$$
\bar{u} c(0, y, z)=Q \delta\left(y-y_{0}\right) \delta\left(z-H_{s}\right)
$$

where now $\mathrm{h}$ is the boundary layer height $(m), \mathrm{L}_{\mathrm{y}}$ is far away from the source, $\mathrm{H}_{\mathrm{s}}$ is the height of the source $(\mathrm{m}), Q$ is the emission rate $(\mathrm{g} / \mathrm{s})$ and $\delta$ is the Dirac delta function.

In order to solve problem (6) by the GILTT method [12, 13], we initially apply the integral transform technique in the y variable. To this end, we expand the pollutant concentration as:

$$
\bar{c}(x, y, z)=\sum_{m=0}^{M} \bar{c}_{m}(x, z) Y_{m}(y)
$$


where $Y_{m}(y)$ are a set of orthogonal eigenfunctions, given by $Y_{m}(y)=$ $\cos \left(\lambda_{m} y\right)$, and $\lambda_{m}=\frac{m \pi}{L_{y}}(\mathrm{~m}=0,1,2, \ldots)$ are respectively the set of eigenvalues.

To determine the unknown coefficient $\bar{c}_{m}(x, z)$ we substitute eqn. (7) in eqn. (6) and after apply the operator $\int_{0}^{L_{y}} Y_{n}(y) d y$. After some algebra, this procedure leads to the set of $\mathrm{M}+1$ two-dimensional diffusion equations:

$$
\bar{u} \frac{\partial \bar{c}_{m}}{\partial x}=\frac{\partial}{\partial z}\left(K_{z} \frac{\partial \bar{c}_{m}}{\partial z}\right)-\frac{\partial}{\partial z}\left(\beta \bar{u} \frac{\partial \bar{c}_{m}}{\partial x}\right)-\lambda_{m}^{2} K_{y} \bar{c}_{m}-\lambda_{m}^{2} K_{y} \frac{\partial}{\partial z}\left(\beta \bar{c}_{m}\right)
$$

The problem (8) is then solved analytically by the GILTT method following the works $[12,22,23]$. We pose the solution of problem (8) in the form:

$$
\bar{c}_{m}(x, z)=\sum_{l=0}^{L} \bar{c}_{m, l}(x) \zeta_{l}(z)
$$

where $\zeta_{l}(z)$ are a set of orthogonal eigenfunctions, given by $\zeta_{l}(z)=\cos \left(\eta_{l} z\right)$, and $\eta_{l}=\frac{i \pi}{h}(1=0,1,2, \ldots)$ are respectively the set of eigenvalues.

Replacing eqn. (9) in eqn. (8) and taking moments, we get the first order matrix differential equation:

$$
\frac{d}{d x} P_{m}(x)+G \cdot P_{m}(x)=0
$$

for $\mathrm{m}=0: \mathrm{M}$, where $P_{m}(x)$ is the column vector whose components are $\bar{c}_{m, l}$ for 1 $=0: L$. The matrix $G$ is defined as $G=B_{1}{ }^{-1} B_{2}$. The entries of matrices $B_{1}$ and $B_{2}$ are, respectively, given by:

$$
\begin{gathered}
\left(b_{1}\right)_{l, j}=-\int_{0}^{h} \bar{u} \zeta_{l}(z) \zeta_{j}(z) d z \\
\left(b_{2}\right)_{l, j}=\int_{0}^{h} K_{z} \zeta_{l}{ }^{\prime}(z) \zeta_{j}(z) d z-\lambda_{l}^{2} \int_{0}^{h} K_{z} \zeta_{l}(z) \zeta_{j}(z) d z-\eta_{l}^{2} \int_{0}^{h} K_{y} \zeta_{l}(z) \zeta_{j}(z) d z
\end{gathered}
$$

Similar procedure leads to the boundary condition of problem (10):

$$
P_{m}(0)=\bar{c}_{m, l}(0)=Q A^{-1} \zeta_{j}\left(H_{s}\right) Y\left(y_{0}\right)
$$

where $A^{-1}$ is the inverse of matrix A having the entry: $a_{l, j}=\int_{0}^{h} \bar{u} \zeta_{l}(z) \zeta_{j}(z) d z$. Likewise the work [12], we solve the problem (10) in a similar manner applying Laplace transform and diagonalization getting

$$
\overline{P_{m}}(s)=X(s I+D)^{-1} \xi
$$

where $\xi\left(\xi=X^{-1} P_{m}(0)\right)$ is found from the equation $X \xi=P_{m}(0)$, and their values are calculated by decomposition $L U$, whose computational cost is smaller than an inversion of matrix. The elements of the matrix (sI+D) have the form $\left\{s+d_{i}\right\}$ where $d_{i}$ are the eigenvalues of the matrix $\mathrm{G}$ and the elements of 
$(s I+D)^{-1}$ are $\frac{1}{s+d_{i}}$ whose transformed inverse of Laplace is $e^{-d_{i} x}$. Let be $\mathrm{G}(\mathrm{x})$ the diagonal matrix whose elements are $e^{-d_{i} x}$ the final solution is then given by:

$$
P_{m}(x)=X G(x) \xi
$$

Finally, using the formula (9) we obtained the solution of the 2D problem, where $\zeta_{l}(z)=\cos \left(\eta_{l} y\right)$ and $\bar{c}_{m, l}(x)$ comes from the solution of the transformed problem given by eqn. (10). Once $\bar{c}_{m}(x, z)$ is known we are in a position to write the final three-dimensional solution of problem (6) which is given by eqn. (7).

This solution is named as 3D-GILTT (three-dimensional GILTT solution).

We promptly realize that the advection-diffusion equation governed by Fickian flow [13] is readily obtained by making the parameter $\beta$, responsible for nonlocal transport, to vanish $(\beta \rightarrow 0)$.

\section{The turbulent parameterization}

The literature reports many, greatly varied formulae, for the calculation of the vertical turbulent diffusion coefficient [14]. As an example of application of our new solution we tested the following vertical and lateral diffusion parameterization suggested by Degrazia et al. [24] for convective conditions:

$$
\begin{gathered}
\frac{K_{z}}{w_{*} h}=0.22\left(\frac{z}{h}\right)^{1 / 3}\left(1-\frac{z}{h}\right)^{1 / 3}\left[1-\exp \left(-\frac{4 z}{h}\right)-0.0003 \exp \left(\frac{8 z}{h}\right)\right] \\
K_{y}=\frac{\sqrt{\pi} \sigma_{v}}{16\left(f_{m}\right)_{v} q_{v}}
\end{gathered}
$$

with $\quad \sigma_{v}^{2}=\frac{0.98 c_{v}}{\left(f_{m}\right)_{v}^{2 / 3}}\left(\frac{\psi_{\varepsilon}}{q_{v}}\right)^{2 / 3}\left(\frac{z}{h}\right)^{2 / 3} w_{*}^{2} ; \psi_{\varepsilon}^{1 / 3}=\left[\left(1-\frac{z}{h}\right)^{2}\left(-\frac{z}{L}\right)^{-2 / 3}+0.75\right]^{1 / 2}$; $q_{v}=4.16 \frac{z}{h} ;\left(f_{m}\right)_{v}=0.16$. More, $k$ is the von Karman constant $(k=0.4), w_{*}$ is the convective velocity scale, $\sigma_{v}$ is the Eulerian standard deviation of the longitudinal turbulent velocity, $q_{\nu}$ is the stability function, $\psi_{\varepsilon}$ is the nondimensional molecular dissipation rate function and $\left(f_{m}\right)_{v}$ is the peak wavelength of the turbulent velocity spectra.

In order to evaluate the vertical wind velocity variance $\sigma_{w}$ and Lagrangian time scale $T_{l w}$ in $\beta=0.55 \mathrm{~S}_{\mathrm{k}} \sigma_{\mathrm{w}} \mathrm{T}_{\mathrm{lw}}$ the following expressions were used [25-27]:

$$
T_{L_{w}}=\frac{0.55}{4} \frac{1}{\sigma_{w}} \frac{z}{\left(f_{m}^{*}\right)_{w}}
$$

where $\left(f_{m}^{*}\right)_{w}=z /\left(\lambda_{m}\right)_{w}$ is the reduced frequency of convective spectral peak and $\left(\lambda_{m}\right)_{w}=1.8 \mathrm{~h}[1-\exp (-4 z / h)-0.0003 \exp (8 z / h)]$ is the peak 
wavelength of the turbulent velocity spectra. For the vertical wind velocity variance:

$$
\sigma_{w}^{2}=1.06 c_{w} \frac{\psi^{2 / 3}}{\left(f_{m}^{*}\right)_{w}^{2 / 3}}\left(\frac{z}{h}\right)^{2 / 3} w_{*}^{2}
$$

where $\psi$ is the molecular dissipation of turbulent velocity [28]:

$$
\psi=1.5-1.2\left(\frac{z}{h}\right)^{1 / 3}
$$

The wind speed profile can be described by a power law expressed as follows [29]:

$$
\frac{\overline{u_{z}}}{\overline{u_{1}}}=\left(\frac{z}{z_{1}}\right)^{n}
$$

where $\overline{u_{z}}$ and $\overline{u_{1}}$ are the mean wind speeds horizontal to heights $\mathrm{z}$ and $\mathrm{z}_{1}$ and $n$ is an exponent that is related to the intensity of turbulence [30].

Thus, in this study we introduce the eddy diffusivities, countergradient expressions and the wind profile described above in the 3D-GILTT model to calculate the ground-level concentration of emissions released from an elevated continuous source point in an unstable ABL.

\section{Application to meteorological scenario}

In order to show the performance of the present solution of the advectiondiffusion equation we have applied the model using the Copenhagen experimental datasets. The Copenhagen field campaign [31] took place in the suburbs of Copenhagen, where $\mathrm{SF}_{6}$ tracer was released without buoyancy from a tower at a height of $115 \mathrm{~m}$ and collected at ground-level on arcs located 2000, 4000 , and 6000 meters from the release point. The site is mainly residential with a roughness length of $0.6 \mathrm{~m}$. The meteorological conditions during the dispersion experiments ranged from moderately unstable to convective. Tracer releases typically started up $1 \mathrm{~h}$ before the tracer sampling and stopped at the end of the sampling period.

We have evaluated the performances of the solutions using the above data sets with the ABL parameterisations presented and for two different values for the skewness: $S_{k}=1$, as suggested by van Dop and Verver [20] and $S_{k}=0.6$, as suggested by Wyngaard and Weil [21].

Figure 1 shows the observed and predicted scatter diagram of centerline ground-level concentrations normalized with the emission source rate $(c / Q)$. This figure shows that a good agreement is obtained between experimental data and the model considering the local and nonlocal closure of turbulence. 


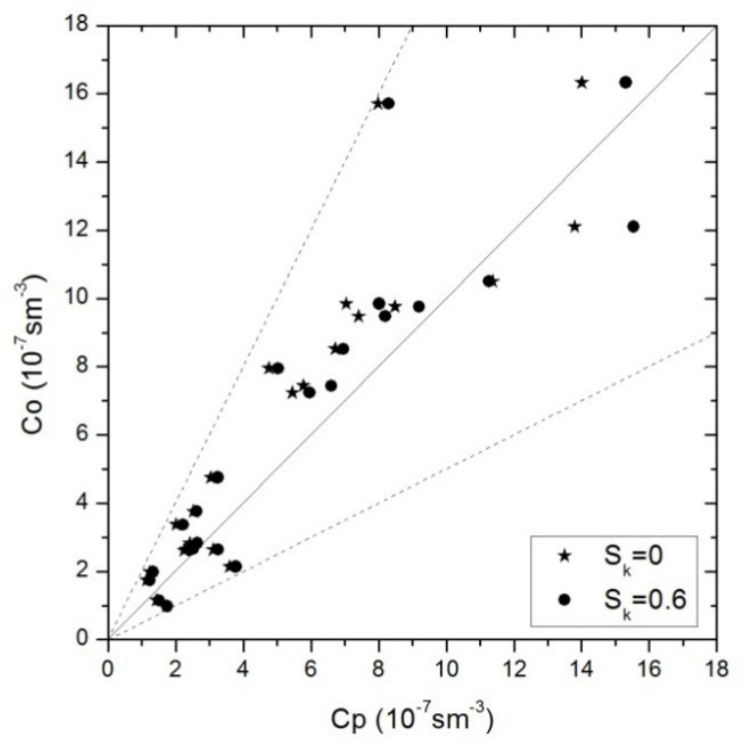

Figure 1: $\quad$ Observed and predicted scatter diagram of ground-level centerline concentrations using the 3D-GILTT approach for the Copenhagen experiment. Dotted lines indicate a factor of two.

Further, we use standard statistical indices in order to compare the quality of the new approach. Table 1 presents some performances evaluations of the model results for the above experiment using the statistical evaluation procedure described by Hanna [32] and defined in the following way:

NMSE (normalized mean square error) $=\overline{\left(C_{o}-C_{p}\right)^{2}} / \overline{C_{p}} \overline{C_{o}}$,

$\mathrm{FA} 2=$ fraction of data $(\%$, normalized to 1$)$ for $0.5 \leq\left(C_{p} / C_{o}\right) \leq 2$,

$\operatorname{COR}$ (correlation coefficient) $=\overline{\left(C_{o}-\overline{C_{o}}\right)\left(C_{p}-\overline{C_{p}}\right)} / \sigma_{o} \sigma_{p}$,

$\mathrm{FB}($ fractional bias $)=\overline{C_{o}}-\overline{C_{p}} / 0.5\left(\overline{C_{o}}+\overline{C_{p}}\right)$,

FS (fractional standard deviations $)=\left(\sigma_{o}-\sigma_{p}\right) / 0.5\left(\sigma_{o}+\sigma_{p}\right)$,

where the subscripts $o$ and $p$ refer to observed and predicted quantities, respectively, and the overbar indicates an averaged value. The statistical index FB says if the predicted quantities underestimate or overestimate the observed ones. The statistical index NMSE represents the model values dispersion in respect to data dispersion. The best results are expected to have values near to zero for the indices NMSE, FB and FS, and near to 1 in the indices COR and FA2. The statistical indices point out that a reasonable agreement is obtained between experimental data and the 3D-GILTT model for Fickian $\left(S_{k}=0\right)$ and non-Fickian cases $\left(S_{k}=0.6\right.$ and $\left.S_{k}=1\right)$. To obtain the results we used L=60 eigenvalues in the $\mathrm{z}$ summation and $\mathrm{M}=10$ in the $\mathrm{y}$ summation. 
Table 1: $\quad$ Statistical results obtained with the 3D-GILTT method compared with the Copenhagen experiment.

\begin{tabular}{|c|c|c|c|c|c|}
\hline 3D-GILTT & NMSE & COR & FA2 & FB & FS \\
\hline$S_{k}=0$ & 0.15 & 0.91 & 1.00 & 0.20 & 0.17 \\
\hline$S_{k}=0.6$ & 0.12 & 0.91 & 1.00 & 0.12 & 0.09 \\
\hline$S_{k}=1$ & 0.14 & 0.88 & 1.00 & 0.08 & -0.01 \\
\hline
\end{tabular}

\section{Conclusions}

We have presented a general solution of the three-dimensional steady-state advection-diffusion equation considering nonlocal turbulence closure, which can be applied in operative models for describing turbulent dispersion of many scalar quantities, such as air pollution, radioactive material, heat and so on. In order to show the performances of the solution in actual scenarios, we introduced some parameterisations of the $\mathrm{ABL}$ and compared the values predicted by the solution with data collected during the well-known Copenhagen experiment. The analysis of the results shows a good agreement between the computed values against the experimental ones.

The differences among the experimental data do not depend on the solution of the diffusion equation, but on the equation itself, which is only a model of reality and on the parameterisation used. Also these results show that, when using models, while they are rather sophisticated instruments that ultimately reflect the current state of knowledge on turbulent transport in the atmosphere, the results they provide are subject to a considerable margin of error. This is due to various factors, including in particular the uncertainty of the intrinsic variability of the atmosphere. Models, in fact, provide values expressed as an average, i.e. a mean value obtained by the repeated performance of many experiments, while the measured concentrations are a single value of the sample to which the ensemble average provided by models refer. This is a general characteristic of the theory of atmospheric turbulence and is a consequence of the statistical approach used in attempting to parameterize the chaotic character of the measured data.

For the above considerations, an analytical solution can be useful in evaluating the performances of numerical method (that solve numerically the advection diffusion equation) that could compare their results, not only against experimental data but, in an easy way, with the solution itself in order to check numerical errors without the uncertainties presented above.

\section{Acknowledgements}

The authors thank Brazilian CNPq and FAPERGS and Italian CNR for the partial financial support of this work. 


\section{References}

[1] Rounds W., Solutions of the two-dimensional diffusion equation. Trans. Am. Geophys. Union 36, pp. 395-405, 1955.

[2] Demuth, C., A contribution to the analytical steady solution of the diffusion equation for line sources. Atmos. Environ. 12, pp. 1255-1258, 1978.

[3] Nieuwstadt F.T.M. \& de Haan B.J., An analytical solution of onedimensional diffusion equation in a non.stationary boundary layer with an application to inversion rise fumigation. Atmos. Environ. 15, pp. 845-851, 1981.

[4] Tirabassi, T., Analytical air pollution and diffusion models. Water, Air and Soil Pollution 47, pp. 19-24, 1989.

[5] Sharan, M., Singh, M.P. \& Yadav, A.K., A mathematical model for the atmospheric dispersion in low winds with eddy diffusivities as linear function of downwind distance. Atmos. Environ. 30, pp. 1137-1145, 1996.

[6] Tirabassi, T., Operational advanced air pollution modeling. PAGEOPH, 160 (1-2), pp. 5-16, 2003.

[7] Costa, C.P., Vilhena, M.T., Moreira, D.M. \& Tirabassi, T., Semi-analytical solution of the steady three-dimensional advection-diffusion equation in the planetary boundary layer. Atmos. Environ. 40 (29), pp. 5659-5669, 2006.

[8] Moreira, D.M, Vilhena, M.T., Tirabassi, T., Costa, C. \& Bodmann, B., Simulation of pollutant dispersion in atmosphere by the Laplace transform: the ADMM approach. Water, Air and Soil Pollution 177, pp. 411-439, 2006a.

[9] Costa, C.P., Tirabassi, T., Vilhena, M.T. \& Moreira, D.M., A general formulation for pollutant dispersion in the atmosphere. J. Eng. Math. 72, pp. 1-15, 2011.

[10] Wortmann, S., Vilhena, M.T., Moreira, D.M. \& Buske, D., A new analytical approach to simulate the pollutant dispersion in the PBL. Atmos. Environ. 39, pp. 2171-2178, 2005.

[11] Moreira, D.M., Vilhena, M.T., Buske, D. \& Tirabassi, T., The GILTT solution of the advection-diffusion equation for an inhomogeneous and nonstationary PBL. Atmos. Environ. 40, pp. 3186-3194, $2006 \mathrm{~b}$.

[12] Moreira, D.M., Vilhena, M.T., Buske, D. \& Tirabassi, T., The state-of-art of the GILTT method to simulate pollutant dispersion in the atmosphere, Atmos. Research, 92, pp. 1-17, 2009.

[13] Buske, D., Vilhena, M.T., Segatto, C.F. \& Quadros, R.S., A General Analytical Solution of the Advection-Diffusion Equation for Fickian Closure. In: Integral Methods in Science and Engineering: Computational and Analytic Aspects. Boston: Birkhäuser, 2011, pp. 25-34.

[14] Seinfeld, J.H. \& Pandis, S.N., Atmospheric chemistry and physics, John Wiley \& Sons, New York, 1998.

[15] Blackadar, A.K., Turbulence and diffusion in the atmosphere: lectures in Environmental Sciences, Springer-Verlag, 1997.

[16] Deardoff, J.W. \& Willis, G.E., A parameterization of diffusion into the mixed layer, J. Appl. Meteor. 14, pp. 1451-1458, 1975. 
[17] Deardoff, J.W., The countergradient heat flux in the lower atmosphere and in the laboratory. J. Atmos. Sci. 23, pp. 503-506, 1966.

[18] Ertel, H., Der vertikale turbulenz-wärmestrom in der atmosphäre. Meteor. Z. 59, pp. 250-253, 1942.

[19] Deardoff, J.W., Numerical investigation of neutral and unstable planetary boundary layers. J. Atmos. Sci. 29, pp. 91-115, 1972.

[20] van Dop, H. and Verver, G., Countergradient transport revisited. J. Atmos. Sci. 58, pp. 2240-2247, 2001.

[21] Wyngaard, J.C. \& Weil, J.C., Transport asymmetry in skewed turbulence. Phys. Fluids A 3, pp. 155-162, 1991.

[22] Buske, D., Vilhena, M.T., Moreira, D.M. \& Tirabassi, T., An analytical solution of the advection-diffusion equation considering non-local turbulence closure. Environ. Fluid Mechanics 7, pp. 43-54, 2007.

[23] Buske, D., Vilhena, M.T., Moreira, D.M. \& Bodmann, B., An analytical solution for the steady-state two-dimensional diffusion-advectiondeposition model by the GILTT approach. In: Integral Methods in Science and Engineering: Techniques and Applications. Boston: Birkhauser, 27-36, 2010.

[24] Degrazia, G.A., Campos Velho, H.F. \& Carvalho, J.C., Nonlocal exchange coefficients for the convective boundary layer derived from spectral properties. Contr. Atmos. Phys. 70, pp. 57-64, 1997.

[25] Degrazia, G.A., Moreira, D.M. \& Vilhena, M.T., Derivation of an eddy diffusivity depending on source distance for vertically inhomogeneous turbulence in a convective boundary layer. J. Appl. Meteor. 40, pp. 12331240, 2001.

[26] Kaimal, J.C., Wyngaard, J.C., Haugen, D.A., Coté, O.R., Izumi, Y., Caughey, S.J. \& Readings, C.J., Turbulence structure in the convective boundary layer. J. Atmos. Sci. 33, pp. 2152-2169, 1976.

[27] Caughey, S.J., Observed characteristics of the atmospheric boundary layer. In: Atmospheric turbulence and air pollution modeling, Boston, 1982.

[28] Druilhet, A., Frangi, J.P, Guedalia, D. \& Fontan, J., Experimental studies of the turbulence structure parameters of the convective boundary layer. $J$. Clim. Appl. Meteorol. 22, pp. 594-608, 1983.

[29] Panofsky, H. A. \& Dutton, J. A., Atmospheric Turbulence, John Wiley \& Sons, New York, 1988.

[30] Irwin, J.S., A theoretical variation of the wind profile power-low exponent as a function of surface roughness and stability, Atmos. Environ., 13, pp. 191-194, 1979.

[31] Gryning, S.E. \& Lyck, E., Atmospheric dispersion from elevated source in an urban area: comparison between tracer experiments and model calculations. J. Appl. Meteor. 23, pp. 651-654, 1984.

[32] Hanna, S.R., Confidence limit for air quality models as estimated by bootstrap and jacknife resampling methods. Atmos. Environ. 23, pp. 13851395, 1989. 